\title{
CARACTERÍSTICAS BIOMÉTRICAS DE MUDAS DE Eucalyptus spp. SOB ESTRESSE SALINO
}

\author{
Andréa V. R. Mendonça', José Geraldo de A. Carneiro², Deborah G. Barroso²,
} Kelly R. Lamônica ${ }^{3}$, Patrícia Ribeiro ${ }^{4}$

\section{RESUMO}

Estudar o comportamento de mudas de Eucalyptus submetidas a estresse salino é fundamental para garantir o sucesso dos plantios em áreas afetadas por sais. $\mathrm{O}$ objetivo deste trabalho foi avaliar o efeito do aumento da concentração de $\mathrm{NaCl}$ sobre características biométricas da parte aérea e sistema radicular de mudas de Eucalyptus camaldulensis, $E$. tereticornis, E. pellita e E. robusta.

O experimento foi conduzido em casa de vegetação, em vasos de capacidade para $11,5 \mathrm{~L}$, contendo areia irrigada com solução nutritiva. $\mathrm{O}$ delineamento experimental foi inteiramente casualizado em esquema fatorial, com quatro espécies e cinco niveis de salinidade $\left(1,41 ; 2,50 ; 4,50 ; 6,45\right.$ e 8,33 dS $\left.\mathrm{m}^{-1}\right)$ com cinco repetições.

A concentração de $\mathrm{NaCl}$ até a condutividade elétrica de $8,33 \mathrm{dS} \mathrm{m}^{-1}$, não comprometeu o desenvolvimento inicial de mudas de $E$. tereticornis. O crescimento das mudas de $E$. camaldulensis, E. pellita e E. robusta foi reduzido em resposta ao aumento do nivel de salinidade.

Palavras-chave: $\mathrm{NaCl}$, sistema radicular, condutividade elétrica, solução nutritiva.

\footnotetext{
'Eng. Florestal Dra. Produção Vegetal. UENF, Av. Alberto Lamego, 2000, 28015-620 - Campos dos Goytacazes-RJ, Brasil: avrmendonca@hotmail.com

${ }_{2}^{2}$ Prof. Silvicultura LFIT/CCTAVUENF: carneiro@uenf.br; deborah@uenf.br

${ }^{3}$ Eng. Agr. Pós-gradudanda em Produçāo Vegetal: krlamonica@bol.com.br

${ }^{4}$ Estudante de Biologia, Universo: patricia_uenfbio@yahoo.com.br
} 


\section{BIOMETRIC CHARACTERISTICS OF Eucalyptus spp. SEEDLINGS IN SALINITY STRESS}

\section{SUMMARY}

To study the behavior of Eucalyptus seedlings submitted to salinity stress is important to assure the success of plantings in salinities areas. The objective of this work was to evaluate the effect of $\mathrm{NaCl}$ concentrations increasing on biometric characteristics of the stem and root system Eucalipts seedlings.

The experiment was carried out in a greenhouse using pots with $11.5 \mathrm{~L}$ of sand irrigated with in nutrient solution. The experimental was a completely randomized and factorial design with four species (Eucalyptus camaldulensis, E. tereticornis, E. pellita and E. robusta) and increasing $\mathrm{NaCl}$ levels $\left(1,41 ; 2,50 ; 4,50 ; 6,45\right.$ e $\left.8,33 \mathrm{dS} \mathrm{m}^{-1}\right)$, with five replications.

The $\mathrm{NaCl}$ concentration up to $8,33 \mathrm{dS} \mathrm{m}^{-1}$ electric conductivity did not promote harm to the initial growth of the $E$. terecicornis seedlings. However growth of the $E$. camaldulensis, $E$. pellita and $E$. robusta seedlings showed lower values in response to increasing salinity level.

Key words: $\mathrm{NaCl}$, root system, eletric conductivity, nutrient solution. 


\section{CARACTERÍSTICAS BIOMÉTRICAS DE PLANTAS DE Eucalyptus spp. BAJO ESTRÉS SALINO}

\section{RESUMEN}

El estudio del comportamiento de plantas de Eucalyptus sometidas a estrés salino es importante para asegurar de plantaciones con estas especies en áreas salinas. El objetivo de este trabajo es evaluar el efecto de concentraciones crecientes de $\mathrm{NaCl}$ en las características biométricas del tronco y el sistema radicular de plantas de eucaliptos.

El experimento fue desarrollado en invernadero, empleando potes de $11,5 \mathrm{~L}$ con arena regada con una solución nutritiva. El diseño experimental fue factorial completamente aleatorizado con cuatro especies (Eucalyptus camaldulensis, E. tereticornis, E. pellita y E. robusta), niveles crecientes de $\mathrm{NaCl}\left(1,41 ; 2,5 ; 4,5 ; 6,45\right.$ y $\left.8,33 \mathrm{dS} \mathrm{m}^{-1}\right)$ y 5 repeticiones.

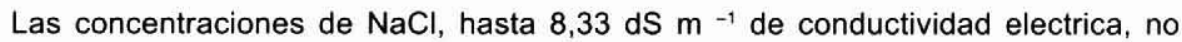
comprometieron el desarrollo inicial de E. tereticornis. Sin embargo, el crecimiento de las plantas de E. camaldulensis, E. pellita y E. robusta, registró valores menores en respuesta a crecientes niveles de salinidad.

Palabras claves: $\mathrm{NaCl}$, salinidad, crecimiento, eucaliptos. 


\section{INTRODUÇÃO}

Para a utilização de solos com excesso de sais, visando produção agrícola ou florestal, é necessária a aplicação de práticas de correção que podem consistir na lixiviação de sais el ou na aplicação de corretivos químicos. Tais práticas envolvem alto custo e não resolvem, efetivamente, o problema. Outras alternativas baseiam-se na utilização de espécies tolerantes à salinidade, tanto para reabilitação do solo como para produção (SU et al., 2005; Mishra et al., 2003; Garg, 1998; Singh et al.,1998; Garg, 1999). Desta maneira, conhecer como mudas de diferentes espécies potenciais respondem à salinidade nas distintas fases de crescimento é importante para garantir o sucesso dos plantios em áreas afetadas por sais.

Este trabalho teve como objetivo avaliar o efeito do aumento da concentração de $\mathrm{NaCl}$ no desempenho inicial de mudas de Eucalyptus camaldulensis, E. tereticornis, E. pellita e E. robusta.

\section{MATERIAL E MÉTODOS}

O experimento foi conduzido no período de maio a novembro de 2004 , em casa de vegetação da Universidade Estadual do Norte Fluminense Darcy Ribeiro (UENF), localizada no município de Campos dos Goytacazes (RJ-Brasil), cujo clima, pela classificação de Köppen, é tropical chuvoso (AW),

O delineamento experimental utilizado foi inteiramente casualizado, em esquema fatorial $4 \times 5$, sendo quatro espécies ( $E$. camaldulensis, E. tereticornis, E. pellita e E. robusta) e cinco níveis de condutividade elétrica $(1,41 ; 2,50 ; 4,50 ; 6,45$ e 8,33 dS m-1), com cinco repetições constituidas por uma muda.

As mudas foram produzidas por três meses e transferidas para vasos com capacidade para $11,5 \mathrm{~L}$, preenchidos com areia lavada. Os vasos foram irrigados com solução nutritiva (Bolles Jones, 1954). Na solução nutritiva foi adicionado $\mathrm{NaCl}$, em diferentes quantidades para obtenção de diferentes niveis de salinidade (Tabela $\mathrm{N}^{\circ} 1$ ).

Tabela $N^{0} 1$

VOLUME DE NACL (2M) NECESSÁRIO PARA PROMOVER OS DIFERENTES NIVEIS DE SALINIDADE PROPOSTOS

\begin{tabular}{|c|c|}
\hline Grau de Salinidade (dS.m- $\mathbf{- 1}^{\mathbf{1}}$ ) & $\mathbf{m l}$ de $\mathbf{N a C l}(\mathbf{2} \mathbf{~ M )}$ por L de solução \\
\hline 1,41 & 0 \\
\hline 2,50 & 5 \\
\hline 4,50 & 15 \\
\hline 6,45 & 25 \\
\hline 8,33 & 35 \\
\hline
\end{tabular}

$\mathrm{Na}$ ocasião do plantio e aos 75 dias após o transplantio foi mensurado diâmetro do colo $(D)$ e a altura $(H)$. Determinou-se a diferença de altura $(H)$ e diâmetro do colo $(D)$ das mudas nas medições realizadas no dia do transplantio e após 75 dias.

Na continuidade das operações, as mudas foram separadas em parte aérea e raízes. 
A parte aérea foi separada em folhas e caules e acondicionadas em sacos de papel. As raizes foram lavadas e classificadas em raiz pivotante + raiz de primeira ordem e raizes a partir da segunda ordem. A massa seca da parte aérea e raiz foi obtida por secagem em estufa a $72^{\circ} \mathrm{C}$, por 48 horas.

Os dados foram submetidos à análise de variância $(\partial=0,05)$ e ao teste Tukey ao mesmo nível de probabilidade (tratamentos de natureza qualitativa) e regressão seqüencial (tratamentos de natureza quantitativa).

\section{RESULTADOS}

O aumento da concentração de $\mathrm{NaCl}$ não influenciou o crescimento em altura (Figura $\mathrm{N}^{\circ}$ 1) e em diâmetro (Figura $\mathrm{N}^{\circ} 2$ ) das mudas de $E$. tereticornis, mas afetou o desenvolvimento das mudas das demais espécies.

$\mathrm{O}$ aumento da concentração de $\mathrm{NaCl}$ não afetou o peso de matéria seca de raizes finas do E. tereticornis e do E. pellita, mas alterou esta característica para as demais espécies, segundo comportamento apresentado na Figura $\mathrm{N}^{\circ} 3$.

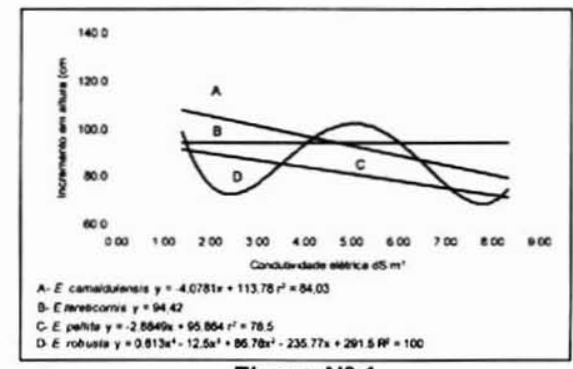

Figura $\mathrm{N}^{\circ} 1$

INCREMENTO EM ALTURA DAS MUDAS DE EUCALIPTO, AOS 75 DIAS APOS

TRANSPLANTIO, EM FUNÇÃO

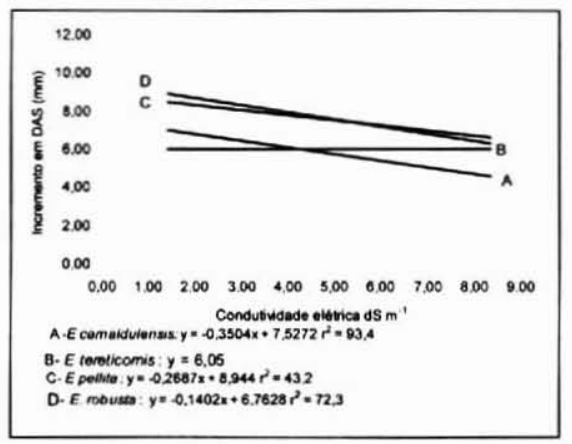

Figura $\mathrm{N}^{\circ} 2$

INCREMENTO EM DIÂMETRO DO COLO (DAS) DAS MUDAS DAS ESPÉCIES DE EUCALIPTO, AOS 75 DIAS APÓS TRANSPLANTIO, EM FUNÇÃO DA CONDUTIVIDADE ELÉTRICA DO SUBSTRATO 


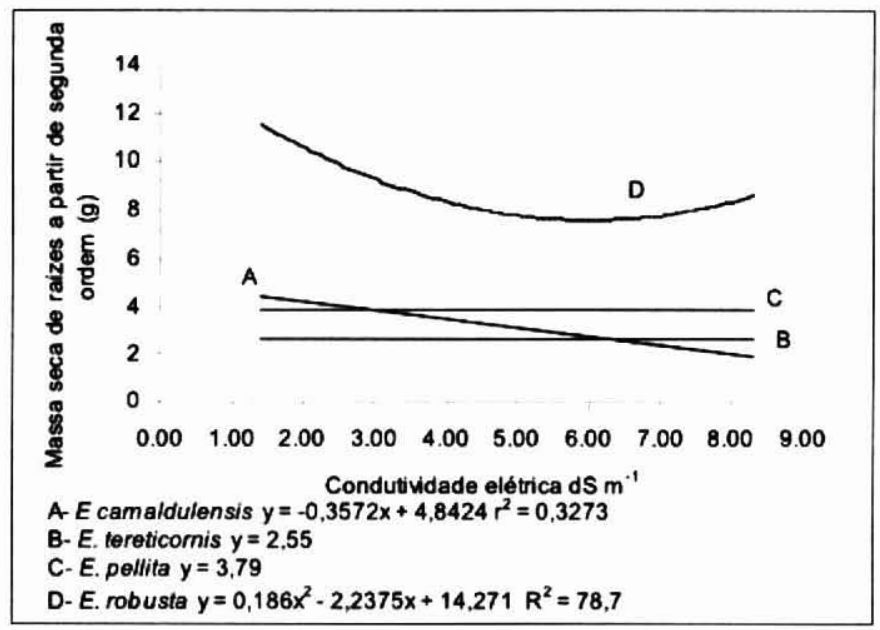

Figura $\mathrm{N}^{\circ} 3$

MASSA SECA DE RAIZZS A PARTIR DA SEGUNDA ORDEM DAS MUDAS DE EUCALIPTO, AOS 75 DIAS APÓS TRANSPLANTIO, EM FUNÇÃO DA CONDUTIVIDADE ELÉTRICA DO SUBSTRATO $(\mathrm{CV} \%=\mathbf{4 7 , 9 )}$

As quatro espécies estudadas não diferiram com relação à massa seca da raiz pivotante+raizes de primeira ordem. Foi detectada uma relação linear simples negativa em resposta ao aumento da condutividade elétrica (Figura $\mathrm{N}^{\circ} 4$ ).

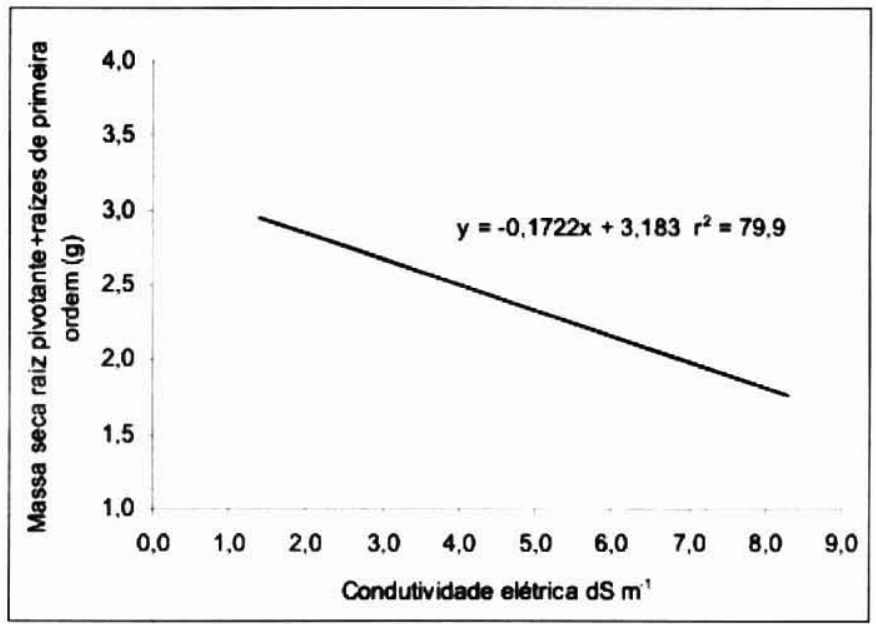

Figura $N^{\circ} 4$

MASSA SECA DE RAIZ PIVOTANTE E DE PRIMEIRA ORDEM DE MUDAS DE EUCALIPTO, AOS 75 DIAS APÓS TRANSPLANTIO, EM FUNÇÃO DA CONDUTIVIDADE ELÉTRICA DO SUBSTRATO $(\mathrm{CV} \%=32,52)$ 
Para as mudas de E. tereticornis a massa seca da parte aérea não foi afetada pela salinidade enquanto que para as demais espécies a salinização causou alterações (Figura $\mathrm{N}^{\circ}$ 5).

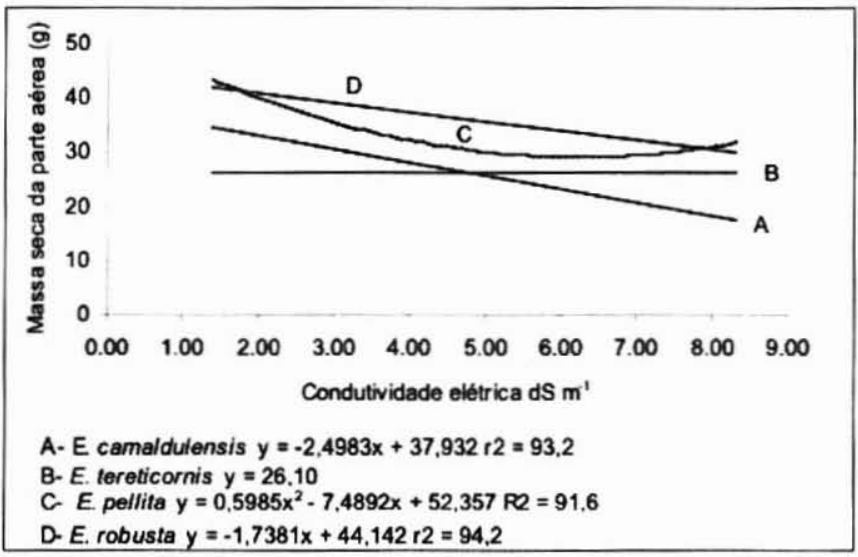

Figura $N^{\circ} 5$

MASSA SECA DA PARTE AÉREA (FOLHA + CAULE) DAS MUDAS DE EUCALIPTO, AOS 75 DIAS APÓS TRANSPLANTIO, EM FUNÇĀO DA CONDUTIVIDADE ELÉTRICA DO SUBSTRATO $(C V \%=20,2)$

O aumento do grau de salinidade influenciou a razão PSA/PSR apenas para as mudas de $E$. pellita, segundo uma relação linear de terceiro grau (Figura $N^{\circ} 6$ ).

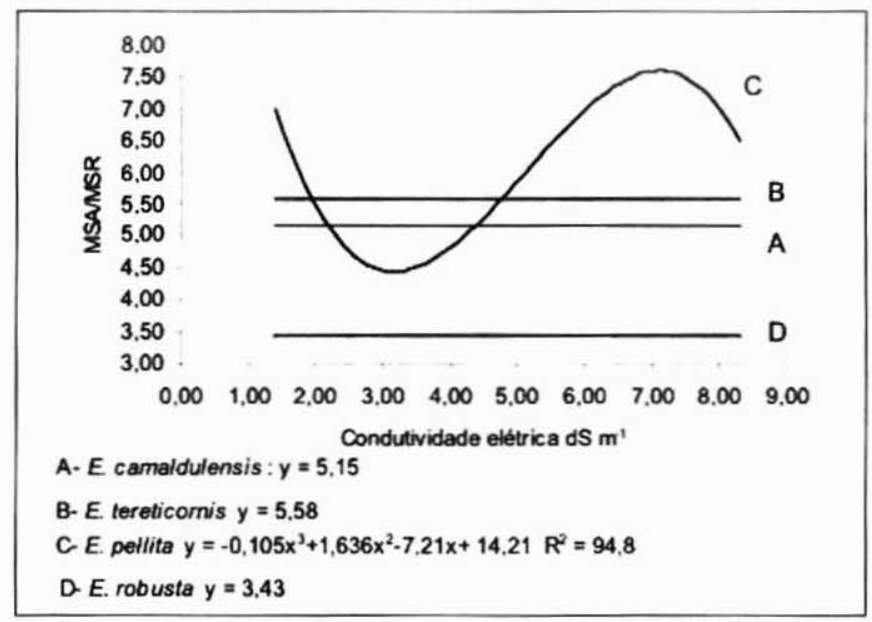

Figura $N^{\circ} 6$

RAZĀO ENTRE MASSA SECA DA PARTE AÉREA E RAIZ (MSAMSR) DE MUDAS DE EUCALIPTO, AOS 75 DIAS APÓS TRANSPLANTIO, EM FUNÇÃO DA CONDUTIVIDADE ELÉTRICA $(C V \%=26,66)$. 


\section{CONCLUSÕES}

A concentração de $\mathrm{NaCl}$ até a condutividade elétrica de $8,33 \mathrm{dS} \mathrm{m}^{-1}$ não comprometeu o desenvolvimento inicial de mudas de E. tereticornis.

O crescimento das mudas de $E$. camaldulensis, E. pellita e E. robusta foi reduzido em resposta ao aumento do nivel de salinidade.

\section{REFERÊNCIAS}

Bolles Jones, E. W., 1954. Nutrition of Hevea brasiliensis I. Experimental methods. Journal of Rubber Research, Institute of Malaysia, v.14, p.183.

Garg, V. K., 1998. Interaction of tree crop with sodic soil environment: Potential for rehabilitation of degraded environments. Land Degradation \& Development, v.9, n.1, p. 81-93.

Garg, V. K., 1999. Leguminous trees for rehabilitation of sodic wasteland in northern India. Restoration Ecology, v. 7, n.3, p. 281-287.

Mishra, A., Sharma, S. D. and Khan, G. H., 2003. Improvement in physical and chemical properties of sodic soil by 3, 6 and 9 yers old plantations of Eucalyptus tereticomis: Biorejuvenation of sodic soil. Forest Ecology and Management, v.184, n. 1-3, p. 115-124.

Singh, G.; Singh, T.; Bhojvaid, P. P., 1998. Amelioration of sodic soils by tree for wheat and oat production. Land Degradation \& Development, v.9, n. 5, p. 453-462.

Su, N., Bethune, M., Mann, L. and Heuperman, A., 2005. Simulating water and salt movement in tile drained fields irrigated with saline water under a Serial Biological Concentration Management Scenario. Agricultural Water Management, v. 78, n. 3, p. 165-180. 